\title{
A Rare Case of Pneumobilia Caused By Ingestion of Methyl Ethyl Ketone Peroxide
}

Ömer TAŞKIN ${ }^{1}$, Ufuk AKDAY², Gökhan SAĞLAMOL ${ }^{3}$, Ayça AÇIKALIN ${ }^{1}$

${ }^{1}$ Çukurova University Faculty of Medicine

${ }^{2}$ Osmaniye Kadirli State Hospital

${ }^{3}$ Kars Harakani State Hospital

\begin{abstract}
Introduction: Methyl Ethyl Ketone Peroxide (MEKP) is a highly toxic substance which is used as a solvent. MEKP causes morbidity and mortality by leading to severe metabolic acidosis, kidney failure and liver failure due to necrosis of the hepatocytes. In this case report we aim to discuss the clinical and radiological findings of an accidental MEKP poisoning.
\end{abstract}

Case Report: A 64-year-old male was referred to our clinic after accidentalingestion of a corrosive substance. The vital signs were normal and fluid treatment was started. The patient was hospitalized after the computerized tomography scan (CT) showed severe esophagitis and pneumobilia. The CT scan revelaed regression on the 3 rd day of hospitalization. The patient was discharged by his own will on the eighth day before providing total recovery.

Conclusion: Unlike other corrosive substances, MEKP may cause intra-abdominal free air such as pneumobilia. An early CT scan helps to evaluate the need for emergency surgical intervention and may prevent patients from unnecessary surgery.

Keywords: Methyl ethyl ketone peroxide, Pneumobilia, Computerized Tomography

\section{Introduction}

Methyl Ethyl Ketone Peroxide (MEKP) is a highly toxic substance which is a clear fluid without any colour. It is used as a solvent, a hardener in the production of resins, synthetic rubber and other petrochemical plastics. It can be drunk by accident because of its water like appearence ${ }^{1}$. MEKP is a strong oxidizer and corrosive agent. The exposure of MEKP leads to free radical formation and this results in lipid peroxidation. The lipid peroxidation may cause dysfunction in cellular level, specially liver and multiple organ failure ${ }^{2}$. MEKP causes liver failure due to necrosis of the hepatocytes, kidney failure, severe metabolic acidosis, edema of the pharynx and larynx, toxic inhalation pneumonitis, corrosive esophagitis, gastrointestinal tract bleeding and hollow organ perforation due to its corrosive effect ${ }^{3,4}$. In the chronic period, it may cause severe strictures on multiple sections of the gastrointestinal tract as well ${ }^{5}$. Gastrointestinal system endoscopy is an important traditional method in the diagnostic staging and treatment of corrosive esophagitis and gastritis.

In this case, we aim to report formation of pneumobilia after exposure of MEKP and to discuss utilization of computed tomography (CT) scan which will be more informative than traditional methods.

\section{Case Report}

A 64-year-old male who is a constructor was referred to our clinic with the complaints of burning in the mouth and back of his sternum, nausea and vomiting after intake of a solvent fluid by accident. The patient applied to our clinic on the third hour of intake. His vitals were stable and in normal limits, the Glaskow Coma Scale score was 15 . The physical examination revealed hyperemia in oropharynx and he had tenderness in epigastrium. His electrocardiyogram (ECG) was normal sinus rhytm. The bottle containing the fluid he had drunk as labelled as Methyl Ethyl Ketone Peroxide (MEKP). His blood tests revealed normal biochemical and hematologic parameters, but metabolic acidosis ( $\mathrm{pH}: 7,23$; pCO2: $45 \mathrm{mmHg}$; hCO3:17 mmol/L). On his chest and abdomen $\mathrm{x}$-ray there was neither sign of perforation nor other pathologies. His oral intake was stopped and daily fluid treatment was started.

After the increase in retrosternal and epigastric pain, he was scanned with intravenous (IV) contrast enhanced abdominal computed tomography (CT). His CT revealed diffuse thick heterogeneous appearance at all levels of esophageal walls and increase in wall thickness. In addition to air densities and para-esophageal fluid densities at the hiatus level near the esophageal wall, air densities (pneumobilia) in intrahepatic biliary tract were also observed (Figure 1). 
After the consultation with the general surgery department, he transferred to the intensive care unit for observation and treatment. The IV contrasted abdominal CT scan of the third day showed regression of all findings (Figure 2). After the regression of his complaints, he discharged from the hospital by his own will.

\section{Discussion}

Common presentations and diagnosis after MEKP intoxications are corrosive esophagitis, gastritis and hepatic necrosis ${ }^{6}$. The traditional diagnostic method of corrosive esophagitis is endoscopy. However, the CT scan is more informative for transmural damage than endoscopy in these patients. The CT imaging provides information about the entire gastrointestinal tract and is very useful in excluding perforation. There are similar CT findings reported in the literature, emphysematous widespread gas formation in the bile ducts and organ walls in the gastrointestinal tract after MEKP exposure ${ }^{6}$. The CT scan is very useful to determine the indication for surgery by the evaluation of gastrointestinal tract, detection of perforation, edema and emphysematous gas formation in the liver and biliary tract.

The gastric decontamination is contraindicated in the patients with MEKP exposure like other corrosive intakes. $\mathrm{N}$-Acetylcysteine is recommended in the patients with acute liver failure and hemodialysis is recommended in the patients with kidney failure ${ }^{6,7}$. To the best of our knowledge of the 30 patients with MEKP exposure reported in the litera- ture, almost all had gastrointestinal truct and liver damage and 10 resulted with mortality ${ }^{5,8}$.

In the case of Jung Oh Chang et al, MEKP exposure had caused gastrointestinal tract damage and liver necrosis ${ }^{6}$. The authors claimed that early endoscopy and CT scan can be used to identify perforation and bleeding in gastrointestinal tract. Endoscopy is diagnostic but CT scan be used to assess the urgent need for surgery. More than 50-100 mililiters (ml) of intake is mortal ${ }^{8,9}$. The drinken amount was nearly $10 \mathrm{ml}$ in our case. Our patient had severe esophagitis in the acute period but acute organ failure did not develop. We believe that the small amount of intake resulted in better response to treatmentandgood outcome.

\section{Conclusion}

MEKP is an agent that may cause death due to multiple organ failure in addition to the corrosive esophagitis. CT imaging in these patients will be more informative than traditional methods and will also help to evaluate the need for emergency surgical intervention.

\section{References}

1. Liyanage IK, Navinan MR, Pathirana AC, Herath HR, Yudhishdran J, Fernandopulle N, et al. A case of methyl ethyl ketone peroxide poisoning and a review of complications and their management. J Occup Med Toxicol. 2015;10:26.

2. Akaike T, Sato K, ljiri S, Miyamoto $Y$, Kohno M, Ando M, et al. Bactericidal activity of alkyl peroxyl radicals generated by

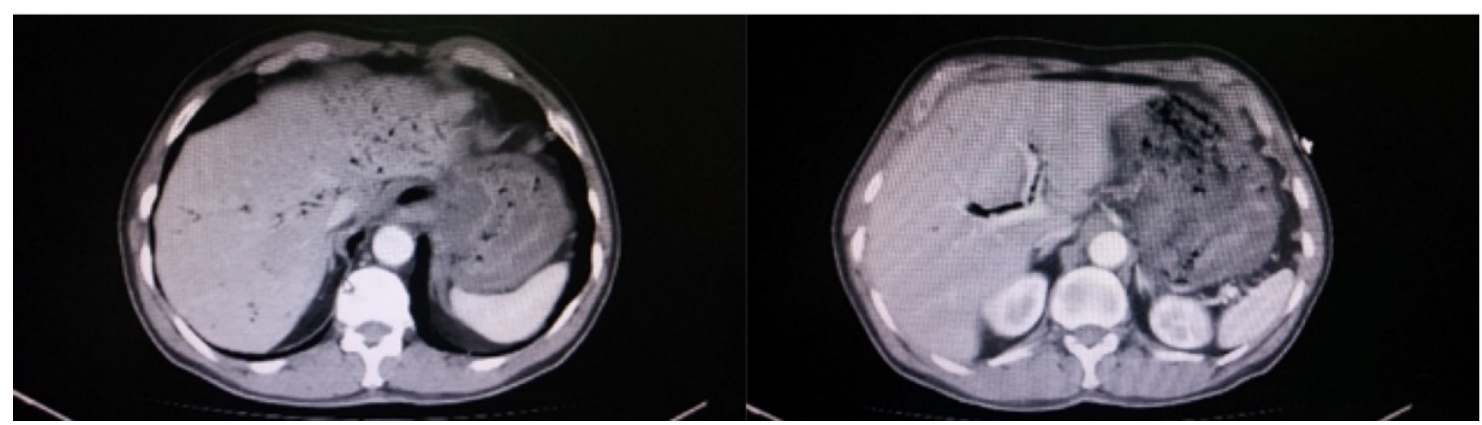

Figure 1: CT image indicating pneumobilia and esophageal wall thickening at day 1 after MEKP accidental oral intake

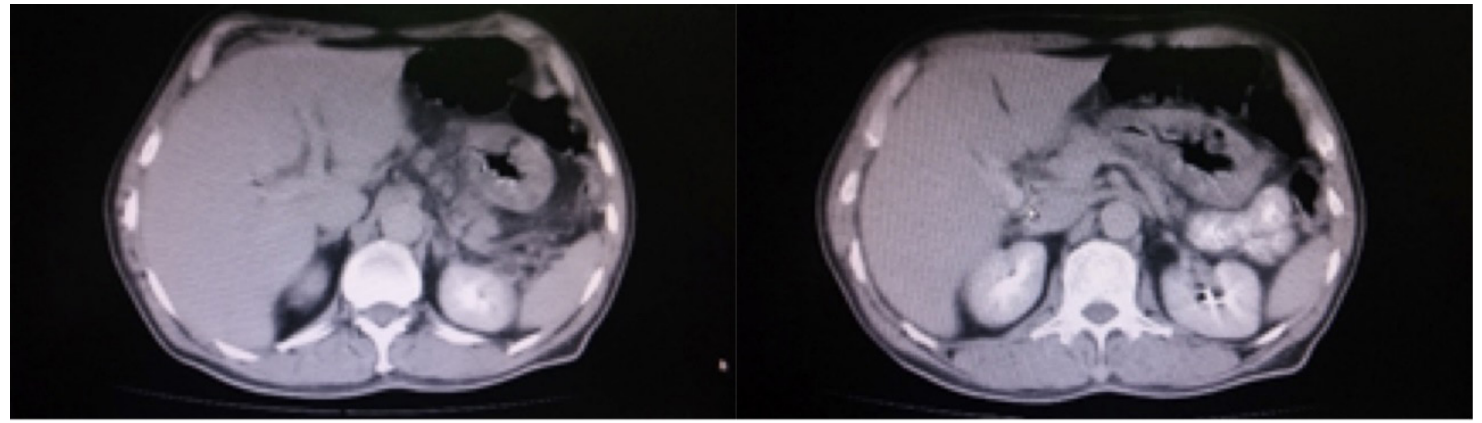

Figure 2: Control CT image at the 3rd day of hospitalization 
heme-iron-catalyzed decomposition of organic peroxides. Archives of biochemistry and biophysics. 1992 Apr;294(1):5563.

3. Moon S-W, Lee S-W, Choi S-H, Hong Y-S. Gastric emphysema after methyl ethyl ketone peroxide ingestion. Clin Toxicol (Phila). 2010 2010/01//;48(1):90-1.

4. Jain AL, Robertson GJ, Rudis MI. Surgical issues in the poisoned patient. Emergency medicine clinics of North America. 2003 Nov;21(4):1117-44.

5. Bates $N$, Driver $C P$, Bianchi A. Methyl ethyl ketone peroxide ingestion: toxicity and outcome in a 6-year-old child. Pediatrics. 2001 Aug;108(2):473-6.

6. Chang JO, Choi JW, Hwang Y. A case of severe corrosive esophagitis, gastritis, and liver necrosis caused by inges- tion of methyl ethyl ketone peroxide. Clin Exp Emerg Med. 2016;3(4):256-61.

7. van Enckevort CCG, Touw DJ, Vleming LJ. N-acetylcysteine and hemodialysis treatment of a severe case of methyl ethyl ketone peroxide intoxication. Clinical Toxicology. 2008 2008/01/01;46(1):74-8.

8. Burger LM, Chandor SB. Fatal ingestion of plastic resin catalyst. Archives of environmental health. 1971 Nov;23(5):402-4.

9. Subbalaxmi MV, Abkari S, Srinivasan VR, Krishnaprasad A. Methyl ethyl ketone peroxide ingestion: a rare cause of corrosive chemical poisoning. The National medical journal of India. 2010 May-Jun;23(3):150-1. 